\title{
THE IMPACT OF DENTURES ON THE NUTRITIONAL HEALTH OF THE ELDERLY
}

\author{
M. Cafaro Gellar, D. Alter
}

\begin{abstract}
Objective: There are many factors that can affect appetite in the older adult. Physiological factors affecting appetite can include cardiovascular disease, pulmonary disease, renal disease, mental health issues, and even side effects of medications. Decreased ability to ambulate due to joint issues or pain can also negatively impact an older adult's appetite. But perhaps one significant factor that is commonly overlooked is the ill fitting partial or complete denture. According to the American Dental Association, there are approximately $57 \%$ of people ages 65 to 74 wearing some form of denture. Due to this large number of denture wearers, it becomes imperative that health care providers learn to incorporate an oral assessment into their plan of care each time an older adult patient is examined. This assessment can assist providers to identify and differentiate unintentional weight loss and loss of appetite as being either part of a disease process or as a symptom of denture issues. Only then can the overall health of the elderly be holistically viewed and treated. The aim of this paper is to provide a summary of published data expressing the nutritional issues that occur in the elderly due to either being edentulous or from wearing improperly fitting dentures.
\end{abstract}

Key words: Elderly, dentures, nutrition, assessment.

\section{Background}

As the baby boomers of this nation and the American population on a whole are aging, certain significance must be applied to evaluating and ensuring proper nutrition and overall physical and mental health. According to the United States Census, there are over 44.7 million adults over the age of 65 as of 2013 (1). The general health of the elderly is negatively impacted by poor nutrition. As a person ages, the body's ability to regulate many functions may become impaired (2). Appetite is controlled by several areas in the body: the gastrointestinal system, the brain, and hormones (3). There is a clear relationship between metabolism and appetite. When a person's metabolism is affected, their appetite is also affected. Due to the inability to move easily, joint pain, or other disease processes, the elderly tend to be less active then when they were younger. This can lead to decreased energy needs which then leads to loss of appetite or anorexia. Any illness can cause a loss of appetite; however, there is not always a definitive cause of anorexia in the elderly, and it may indeed be the first symptom of an undiagnosed illness. Since loss of appetite can cause unintentional weight loss and

New York City College of Technology, City University of New York, USA

Corresponding Author: Michelle Cafaro Gellar, 300 Jay St, Pearl 505, Brooklyn, NY 11209 USA, mgellar@citytech.cuny.edu malnutrition, it is imperative that this symptom not be dismissed as simply a normal part of the aging process. Loss of appetite and malnutrition can also be caused by cancers, cardiovascular disease, pulmonary disease, neurological disease, liver disease, renal failure, and even side effects of medications (3). Mental health issues such as depression, dementia, anxiety and grief may also lead to malnutrition. Factors such as decreased sense of taste, smell and sight dull with age and can also cause a loss of appetite. Additionally, nutritional status impacts on the development of the teeth and an individual's resistance to many oral conditions, including periodontal diseases and oral cancer (4). In light of this knowledge, it now becomes imperative that health care providers establish whether the loss of appetite in an elderly individual is due to a physiological reason, a mental health issue, or a complication of ill fitting dentures.

\section{Denture Use}

It is well established that a good diet is essential for the development and maintenance of healthy teeth, but healthy teeth are important in enabling the consumption of a varied and healthy diet throughout the life cycle (4). The necessities of dental clients, in particular geriatric dental clients, have steadily increased and dental services involving the number of individuals needing 
complete dentures is on the rise. It is estimated that a person reaching 65 will live an additional 17.8 years. A census collected by the American Dental Association has established that nearly $57 \%$ of people ages $65-74$ are wearing some form of denture, either partial or complete (5). As per Douglass, there are currently over 32 million people in the United States wearing partial or complete dentures (5). According to a study performed in 2013 by iData Research Inc., there were 2,822,589 complete dentures and 3,722,183 partial dentures fabricated for American patients, totaling 6,544,772; this reflects an increase of $3.5 \%$ from the previous year (6). Ensuring the validity and proper use of the dental prosthesis or appliance is of paramount importance for the individual's overall health.

Multiple reasons may affect an individual's aptitude in wearing their dental prosthesis. One attribute is the minimization of taste and texture sensation due to covering of palate (7). More significantly, many denture wearers develop painful sores because of ill fitting dental prostheses. Complete and partial dentures must go through a regimented dental protocol to ensure appropriate fit from the onset both in the clinical setting as well as in the laboratory. A poorly fabricated denture could cause harm and discontent for the individual which can potentially lead to cessation of use of the appliance (8). Approximately $33 \%$ of denture wearers have reported their dentures as poor fitting and those individuals were more likely to remove or disuse their dentures while eating (9). Furthermore, a responsible regiment of dental services must be engaged. Adults who wear dentures are required to see a dentist at the minimum of once a year for functioning dentures and more frequently for those with a newer dental prosthesis. The dentures should be removed daily for proper hygiene and to allow the gums to rest. Avoiding these measures would lead to movement in the oral cavity and ultimately deem the dental prosthesis to be ill fitting (7). Those individuals with ill fitting dentures self-reported a significantly lower use of professional dental services, higher degree of oral function limitations, and significantly increased levels of poor health and depression (8).

\section{Assessment}

The Mini-Nutritional Assessment is one tool that is utilized internationally in various healthcare settings to perform a quick yet valid assessment of the nutritional status in the elderly (10). This tool identifies at-risk individuals as well as those who already suffer from malnutrition. Additionally, nutritional status may be measured by Body Mass Index (BMI), serum albumin levels, and self-report of appetite and weight loss. In a study performed by Sheiham and Steele, et. al, the likelihood of older adults having a BMI within the normal range of 20-25 was increased in those who had more than
20 natural teeth. Conversely, adults over 65 years of age who have few natural teeth or no teeth at all were found to be at a greater risk of being underweight due to functional issues leading to inadequate dietary intake, and yet also at a greater risk of being obese due to poor quality of diet $(11,12)$.

Inspection of the oral cavity can provide information regarding dryness of the oral mucosa which can indicate if the individual is experiencing decreased saliva, and can also alert the healthcare provider to evidence of poor oral hygiene. However, while only a small number of the denture wearers studied by Donini reported that decreased saliva caused discomfort while wearing dentures, approximately $30 \%$ of all 65 year olds have been found to have decreased saliva, or xerostomia (13). Xerostomia can lead to problems with chewing and swallowing; therefore, when coupled with poor oral hygiene, xerostomia can lead to changes in dietary intake which can cause malnutrition and involuntary weight loss among frail elderly adults. The ability to chew and digest food may be impaired in the elderly either by the loss of teeth or due to the use of dentures. Impaired dietary intakes were partly associated with poor fitting dentures, lack of teeth or lack of saliva (14). Reduced mastication ability may also lead to a change in the types of foods eaten due to a change in the ability to break down the food or the individual's perception that such changes are necessary.

\section{Nutrient Intake}

The elderly need to eat a variety of nutrient dense foods which can be found in detail on MyPlate (15). This includes a variety of fresh or frozen vegetables, fruit, whole grains, lean protein and adequate water intake. An increase in calcium and vitamin D are needed to maintain bone health as people age, and fiber should be increased as well since peristalsis can slow during the aging process. However, edentulous older adults were found to consume less food energy and significantly less protein, intrinsic and milk sugars, non-starch polysaccharide (fibre), calcium, non-haem iron, niacin and vitamin C than dentate people (14). A Tufts University study of older adults found that full or partial denture wearers had diets considerably lower in 19 different nutrients as compared to adults without missing teeth (7). Many of the nutrients missing are found in hard to chew foods such as stringy meats, some vegetables and fruits such as carrots and apples, as well as nuts. Diets high in fat and low in fiber by edentulous individuals could be due to reduced mastication ability in those wearing full replacement dentures (16). Individuals with coexisting health issues or disabilities may be more vulnerable to reduced nutrient intake (14). Another factor affecting nutrition in adults is food preference, which is usually dictated by socio-cultural background as well as economic status, educational level and dwelling 
type. When individuals cannot prepare meals for themselves, they usually do not have control over the choice of foods included in their diet. This is also true for older adults who reside in various types of institutions. These factors need to be further studied in relation to the cause of malnutrition in the elderly since better fitting appliances or replacement of ill fitting appliances may not make a difference in nutritional intake. Therefore, involuntary weight loss may be caused by a conscious change in dietary intake and food avoidances as opposed to changes in mastication from dentures.

\section{Discussion}

Older Americans who currently have poor oral health have been disadvantaged by not having fluoridated water and oral hygiene products containing fluoride in their younger years. The risk for poor oral health also increases among those who are socio-economically disadvantaged, those who live in rural areas, lack dental insurance, are disabled, homebound, or institutionalized (8). About $25 \%$ of those 40.3 million older adults no longer have any natural teeth (1). One reason why the elderly do not pursue dental care is related to lack of dental insurance. Adults over the age of 65 who qualify for Medicare are provided with medical coverage but without dental coverage for any type of oral care (8). Older adults who qualify for Medicaid from the federal government will have dental coverage but reimbursements are low and therefore providers may be more difficult to find. Access to oral health care is one of the greatest disparities in the United States today based on ethnicity and socio-economic status. As a result, the treatment, management, and prevention of oral diseases in the elderly will improve not only the conditions of their mouths, but also their overall health and wellbeing (17). Research has proven that dental disease can contribute to morbidity and mortality in older adults as well as a decreased quality of life in this population.

Poor oral health may also lead to social isolation due to embarrassment from odor and appearance. Although oral co-morbidities are common in older adults, their association with medical and functional status is often neglected during the geriatric assessment (8). Geriatricians and family practitioners need to start performing an oral evaluation in addition to their standard assessments when the patient is an older adult, as suggested in Table 1. Adults with full dentures need to continue with their scheduled oral assessments as well. Dentures may need alterations over time, and can cause abrasions and edema of the gums. Difficulties in chewing, discomforts with dentures as well as ill fitting dentures of poor quality are common in old age. These complications may ultimately lead the individual wearing the appliances to drastically decrease or alter their oral intake due to pain or discomfort. However, there does seem to be a level of functional discomfort that denture-wearers seem to be willing to cope with, according to Altenhoevel, et al. (18) Attention to the oral cavity may improve the quality of life for the older adult and decrease the risk for other comorbidities while preventing the individual from being at risk for poor diet and nutritional insufficiencies. Proper fitting dental prostheses produced from quality materials is of equal importance for the older adult's overall quality of life.

Table 1

Suggested guidelines for healthcare providers

In addition to assessing past medical/surgical history:

Perform:

--Mini Nutritional Assessment

Assess:

--Weight loss/gain, intentional or unintentional

--Alterations in dietary pattern

--Change in types of foods eaten

--Difficulty chewing, change in taste

Inspect oral cavity for:

--Full or partial dentures

--Assess for dry mucosa

--Sores, abrasions, irritations

Follow up care:

--Frequency of dental visits

--Referrals needed for dental care or nutritionist

\section{Conclusions}

Dietary intervention and advice for dental health should be focused on health promotion and should follow the guidelines for general health (19). It should be based on each individual patient's dietary capabilities and include the consumption of a variety of healthy foods. Health care professionals need to remain consistent in the advice they provide to clients regarding dietary intake. Oral health should not be viewed as separate from general health. Dietary advice that protects against major health conditions can also lead to dental health. Maintaining natural dentition can ensure adequate masticatory function, which can lead to the ingestion of necessary nutrients in the older adult. While dental function is not the only factor influencing food choice, the value of good teeth for enabling the consumption of a varied diet for enjoyment of food and food-related quality of life is an important consideration for nutrition and dental health professionals (4).

Ethical Standards: Neither author has any conflict of interest with review and summation of the information gathered.

Conflicts of Interest: The authors declare that there are no conflicts of interest. 


\section{References}

1. Werner C. The older population: 2010. U.S. Dept. of Commerce, Economics and Statistics Administration, U.S. Census Bureau.http://www.census.gov/ prod/cen2010/briefs/c2010br-09.pdf. Published 2011. Accessed February 8, 2013.

2. Mangoni A, Jackson S. Age-related changes in pharmacokinetics and pharmacodynamics: basic principles and practical applications. BritishJournal of Clinical Pharmacology [serial online]. 2004;57(1):6-14. Available from: CINAHL Plus with Full Text, Ipswich, MA. Accessed February 8, 2013.

3. Donini L, Savina C, and Cannella C. Eating habits and appetite control in the elderly: the anorexia of aging. International Psychogeriatrics[serial online]. 2003;15(1): 73-87. Available from: CINAHL Plus with Full Text, Ipswich, MA. Accessed February 8, 2013.

4. Moynihan, Paula. «The Interrelationship between Diet and Oral Health.» Proceedings of the Nutrition Society 64.04 (2005): 571-80. Web. 29 Dec. 2014

5. Douglass CW, and Watson AJ. Future needs for fixed and removable partial dentures in the United States. The Journal of Prosthetic Dentistry [serial online]. 2002;87(1): 9-14. Available from: CINAHL Plus with Full Text, Ipswich, MA. Accessed February 8, 2013.

6. IData Research, Inc., n.d. Web. 8 Feb. 2013. <http://www.idataresearch. net.>.

7. Denture Wearers at Risk for Poor Nutrition. Tufts University Health \& Nutrition Letter[serial online]. July 2000;18(5):3. Available from:CINAHL Plus with Full Text, Ipswich, MA.Accessed February 2, 2013.

8. Weyant R, Pandav R, Plowman J, and Ganguli M. Medical and cognitive correlates of denture wearing in older community-dwelling adults.Journal of the American Geriatrics Society[serial online]. April 2004;52(4): 596-600. Available from: CINAHL Plus with Full Text, Ipswich, MA. Accessed on February 7, 2013.

9. Savoca M, Arcury T, Quandt S, et al. Impact of denture usage patterns on dietary quality and food avoidance among older adults. Journal of Nutrition In Gerontology \& Geriatrics [serial online]. January 2011;30(1):86-102. Available from: CINAHL Plus with Text, Ipswich, MA. Accessed February 8, 2013.

10. Soini $\mathrm{H}$, Routasalo $\mathrm{P}$, and Lagström H. Characteristics of the mini-nutritional assessment in elderly home-care patients. European Journal of Clinical Nutrition[serial online]. 2004;58(1): 64-70. Available from: CINAHL Plus with Full Text, Ipswich, MA. Accessed February 7, 2013.

11. Kanehisa Y, Yoshida M, Taji T, Akagawa Y, and Nakamura H. Body weight and serum albumin change after prosthodontic treatment among institutionalized elderly in a long-term care geriatric hospital. Community Dentistry and Oral Epidemiology[serial online]. December 2009; 37(6): 5348. Available from: CINAHL Plus with Full Text, Ipswich, MA. Accessed February 8, 2013

12. Sheiham, A., J.g. Steele, W. Marcenes, S. Finch, and A.w. G. Walls. «The Relationship between Oral Health Status and Body Mass Index among Older People: A National Survey of Older People in Great Britain.» British Dental Journal 192.12 (2002): 703-06. Web. 30 Dec. 2014

13. Bissett S, Preshaw P. Guide to Providing Mouth Care for Older People. Nursing Older People [serial online]. December 2011;23(10):14-21. Available from: CINAHL Plus with Full Text, Ipswich, MA. Accessed February 20, 2013.

14. Sheiham, A., and J. Steele. «Does the Condition of the Mouth and Teeth Affect the Ability to Eat Certain Foods, Nutrient and Dietary Intake and Nutritional Status amongst Older People?» Public Health Nutrition 4.03 (2001): 797. Web. 29 Dec. 2014.

15. «ChooseMyPlate.gov.» ChooseMyPlate.gov. U.S. Department of Agriculture, n.d. Web. 3 Feb. 2013. <http: / / www.choosemyplate.gov/>.

16. Allen P. Association between diet, social resources and oral health related quality of life in edentulous patients. Journal of Oral Rehabilitation[serial online]. September 2005;32(9): 623-628. Available from: CINAHL Plus with Full Text, Ipswich, MA. Accessed February 7, 2013.

17. Ahluwalia K. Editors Choice. Oral health care for the elderly: more than just dentures. American Journal of Public Health [serial online]. May 2004;94(5):698. Available from:CINAHL Plus with Full Text, Ipswich, MA. Accessed on February 8, 2013.

18. Altenhoevel, A., K. Norman, C. Smoliner, and I. Peroz. «The Impact of Self-Perceived Masticatory Function on Nutrition and Gastrointestinal Complaints in the Elderly.» The Journal of Nutrition, Health \& Aging 16.2 (2012): 175-78. Web. 28 Dec. 2014

19. Moynihan, P. J. «Dietary Advice in Dental Practice.» British Dental Journal 193.10 (2002): 563-68. Web. 28 Dec. 2014.

20. Duffin C. Brushing up on oral hygiene. Nursing Older People[serial online]. March 2008; 20(2):14-6. Available from: CINAHL Plus with Full Text, Ipswich, MA. Accessed February 8, 2013.

21. Stein, P. S., \& Henry, R. G. Poor oral hygiene in long-term care: nurses must provide better oral care to older adults and patients with severe disabilities. American Journal of Nursing[serial online]. June 2009; 109(6) 44-50. Available from: CINAHL Plus with Full Text, Ipswich, MA. Accessed February 7, 2013. 